\title{
Composite Reliability Evaluation Combining Adequacy and Security Aspects
}

\author{
Ivo Cristiano Costa ${ }^{1}$ \\ Mauro Augusto da Rosa ${ }^{1}$ \\ Jean Sumaili ${ }^{1}$ \\ ${ }^{1}$ INESC TEC (formerly INESC Porto) \\ Porto, Portugal \\ marosa@inescporto.pt
}

\author{
Leonel de Magalhães Carvalho ${ }^{1,2}$ \\ Diego Issicaba ${ }^{1,2}$ \\ ${ }^{2}$ FEUP, Faculty of Engineering of the University of Porto \\ Porto, Portugal \\ carvalho.leonel@,fe.up.pt
}

\begin{abstract}
Currently, it is an ordinary procedure to restrict the use of renewables, mainly wind power, to accommodate a more stable set of generating units (e.g.: thermal units) to supply the load. The purpose of this paper is to discuss particular aspects from the adequacy and security evaluation of generation and transmission (G\&T) system that must be revised due to the massive integration of wind power in the present and future energy systems. By running a sequential Monte Carlo simulation (SMCS) algorithm it is possible to assess the system adequacy identifying possible security constrains. The demonstration cases are based on the modified IEEE-RTS 79 system and a small planned configuration for a real G\&T system, typically radial. The results extracted from the case studies illustrate that the impact resulting from the massive integration of renewable sources should not be assessed from the adequacy evaluation perspective without considering system security issues.
\end{abstract}

Index Terms-- Power system reliability, Wind power generation, Adequacy and security evaluation.

\section{INTRODUCTION}

Aspects from adequacy and security of power system analysis must be revised due to the integration of massive wind power into present and future electric energy systems. It is well known that the design characteristics of conventional hydro and thermal generators are prepared not only to add capacity into the system, but also to provide conditions to support the system services, such as through voltage and frequency regulation. These design characteristics sustained the idea behind adequacy evaluation assumptions, where stability concerns might be neglected, mainly because system support services were inherent to the generating units. Furthermore, it is also recognized that the design characteristics of a wind turbine are based on a different technology and, at the moment, it is accomplished to provide capacity to the system, but it is not capable of providing the same system support services. This inability weakens the assumption where stability concerns are neglected, mainly because the addition of capacity without considering stability

The work of Leonel de Magalhães Carvalho, Diego Issicaba and Jean Sumaili was supported by Fundação para a Ciência e Tecnologia (FCT) - Ph.D. Scholarships SFRH/BD/43004/2008 and SFRH/BD/ 43049/2008 - and within "Ciência2008" program. characteristics may lead to a wrong evaluation and dangerous conclusions.

Despite the possible techniques and methods that can be applied to evaluate a power system performance, the assumptions used in the past were based on some adequacy and security definitions such as: a) Adequacy evaluation relates to the ability of the installed generation and transmission to serve the total system requirements; b) Security-constrained adequacy evaluation relates to the ability of the generation and transmission system to avoid load curtailment under failure events; c) Security evaluation relates to the ability of the system to operate under stable conditions when a major change in the system occurs [1]. The integrated evaluation of adequacy and security aspects is a nonconsolidated topic in power system analysis. However, it is of a general consensus that increasing the participation of wind power (considering the current technology) in the total generation mix means operating and planning methodologies, and also standards must be revised.

The purpose of this paper is to study the accommodation of huge amounts of wind power without neglecting the consequences that wind turbines may bring to the system security. For this to be accomplished, a SMCS [2] tool is addressed, considering security aspects and exploiting a set of new reliability metrics to be used together with the conventional indices.

\section{Sequential Monte Carlo Simulation}

Widely used, the Monte Carlo simulation methods are one of the most important mechanisms applied for assessing the adequacy of the composite systems. It allows a flexible modeling of the power system components and operational constraints, reproducing the chronological steps of the system operation. The SMCS creates several samples that represent a type of synthetic history of the system operating states, sampling manifold chronological events such as the temporal variation of the primary energy resources as well as load diagrams variations and maintenance programs. In addition, it offers enough flexibility to model all components using 
Markovian and non-Markovian models. By the end of the algorithm, traditional loss of load indices can be estimated, as well as several other distributional aspects linked to these indices can be evaluated [3].

\section{A. Models and Assumptions}

With the purpose of setting the capacity of a component and the generation/load variations, two models were considered: 1) the failure/repair cycle stochastic model and 2) the capacity time-dependent model. The impact of each one of these models, either to define the generation capacity or the load value, is illustrated in Fig. 1, where the two models are proportionally displayed.

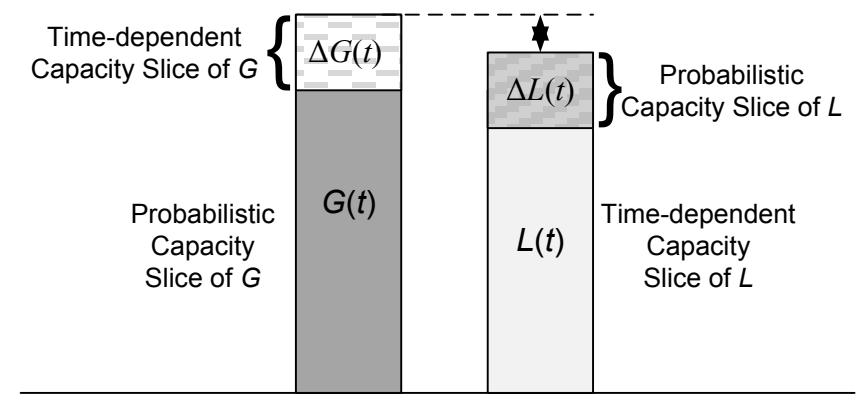

Figure 1. Description of the models for generation/load variations.

The random variable $G$ depends on the equipment availabilities, which is usually modeled supported by the two states and/or multi-states Markov models [4], [5]. At this point, there is no need for chronology representation in the arrangement of the generating system capacity represented by random variable $G$. When the capacity fluctuation effects are modeled, it is necessary to consider time-dependent characteristics of each representation. For instance, reservoir operation rules in hydroelectric systems are strongly linked to the hourly operation decisions and consequently a chronological representation is needed. Before composing the system capacity represented by the random variable $G$, it is necessary to decouple the random variable $G$ into two capacity slices (see Fig. 1) such as, stochastic capacity slice, which is only linked to the stochastic behavior of each component, and time-dependent capacity slice of $G$, which can capture timedependent variations, which was represented as $\Delta G$ in Fig. 1.

The random variable $L$ is composed essentially by hourly observations of capacities. Although, it might be interpreted as a capacity with probabilistic nature, it differs from the assumptions discussed for random variable $G$. In fact, a large slice of this capacity can be classified as time-dependent, whereas the probabilistic capacity slice of $L$, might depends, for instance, on the short- and long-term uncertainties of load representations, which can also be included in any hourly load model, represented by $\Delta L$ in Fig. 1 .

As it can be seen, the power balance equation commonly used on generating adequacy evaluation is composed by different slices of capacities, where the random variables $G$ and $L$ sometimes depends on an entirely stochastic process that does not carry any time-dependent characteristic (e.g. thermal generating outage units or transmission system equipment), and some other times of an entirely timedependent characteristic affected by the stochastic process. Thus, it is possible to decouple $G$ and $L$ representations on several slices of capacities in accordance with each generation technology in order to better represent the power system components behavior. These concepts were extended by G\&T reliability evaluation in this work.

In this study two stochastic representations are effectively used: the two-state [4] and the multistate [5] Markov models. If the component follows the two-state Markov model (Fig. 2), its capacity is only available in the up state.

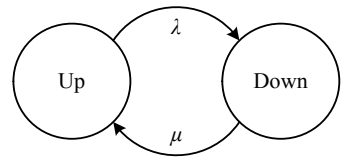

Figure 2. Two-state Markov model ( $\lambda$ is the failure rate and $\mu$ is the repair rate).

However, if the multistate Markov model is used for an aggregation of $N$ components, the representation of the failure/repair cycle is represented as in Fig 3.

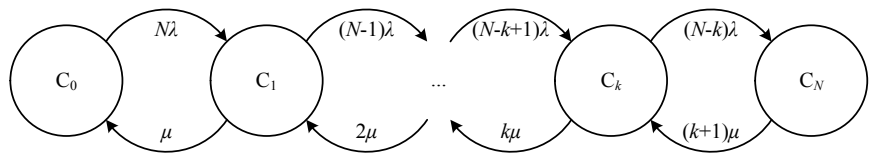

Figure 3. Multistate Markov model

( $\lambda$ is the failure rate, $\mu$ is the repair rate, $N$ is the number of components of the aggregation, $\mathrm{C}_{k}$ is a state which contains $N-k$ components in the $u p$ state).

Concerning the capacity time-dependent model, the capacity of the generating units can vary according with hourly, weekly, monthly or even annually series with probabilities associated, acquired over several years of observation. In Fig.4, the monthly series for hydro and wind power are depicted. These series belong to the IEEE-RTS 96 HW system proposed in [5] and extended to the IEEE-RTS 79 HW in this work.
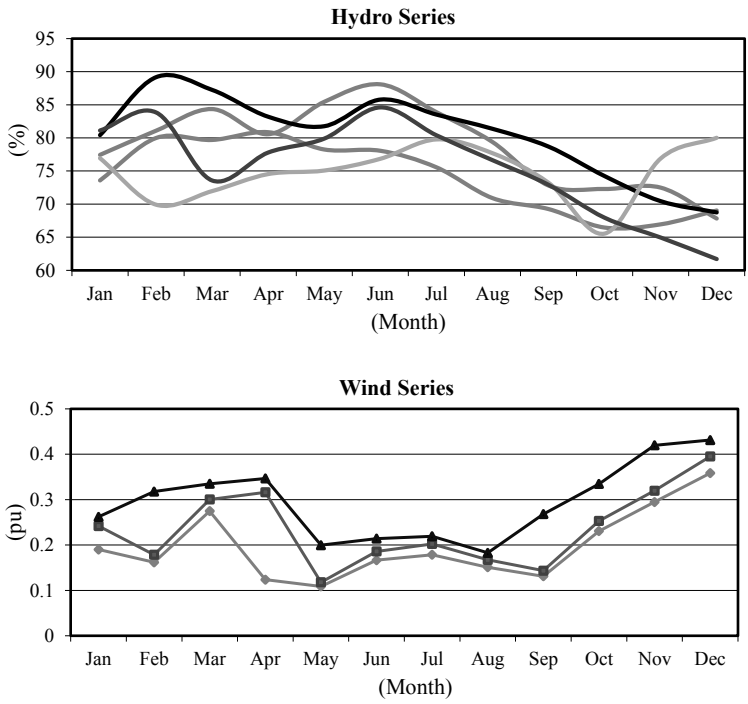

Figure 4. Hydro and Wind Series for the IEEE-RTS $96 \mathrm{HW}$ system. 
For an appropriate capacity model of a certain component, its maximum capacity, which is defined by the failure/repair cycle stochastic model, must be affected by its time-varying characteristic.

\section{B. Component's Models}

For every component a short description of the failure/repair cycle stochastic model and the capacity timedependent model is prepared. These definitions are common for both demonstration tests (IEEE-RTS $79 \mathrm{HW}$ and Real Case Study) excepted when noted.

1) Conventional Generation Units: The failure/repair cycle is represented by a two-state Markov model, with transitions that follow an exponential probability distribution. These generating units do not depend on any time-dependent model since, when in the up state, the conventional generator is able to provide its maximum capacity and in the down state, the generator capacity is zero.

2) Hydro Generation Units without pumping capacity: The failure/repair cycle is represented by a two-state Markov model, with transitions following an exponential probability distribution. The capacity time-dependent model for these units considers a proportional relationship between the total water stored in a reservoir and the total power produced by the corresponding hydro generator. For the IEEE-RTS $79 \mathrm{HW}$ system this proportional relation is defined for each month of the year, based on historical observations, while in the Real Case Study these observations are on hourly basis.

3) Hydro Generation Units with pumping capacity: These units are only available in the Real Case Study. The failure/repair cycle is represented by a two-state Markov model, with transitions that follow an exponential probability distribution. For the time dependency characterization it is assumed that these units adopt a condition of power consumption by pumping during established periods, which may coincide with the off-peak period (between 0 and 6 hours). The purpose for this representation is to emulate the consumption process of storing renewable energy, enabling the reduction of renewable waste.

4) Wind and Solar Farms: Both wind and solar farms consist in the aggregations of small units (wind turbines and photovoltaic panels respectively) that traditionally have the same electrical parameters. Taking advantage of this characteristic, the multistate Markov model is used to describe the failure/repair cycle of the total park, with transitions following an exponential probability distribution. Like hydro units, the capacity time-dependent model considers several annual series with hourly resolution.

5) Transformers and Transmission Lines: The failure/repair cycle for transmission lines and transformers is modeled by a two-state Markov model, with transitions that follow an exponential probability distribution.

6) Loads: The load is modeled using a chronological representation for each bus load, containing 8760 observations.
7) Generator/Transformer Aggregation: Only considered for the Real Case Study. Sometimes the connection of the generating unit with the grid is made through one or more transformers in parallel, as illustrated in Fig. 5a). In this case, if the failure/repair cycle of the unit follows the two-state Markov model, it is possible to create a simplified model for the failure/repair cycle of the generator/transformer aggregation (Fig. 5b)).

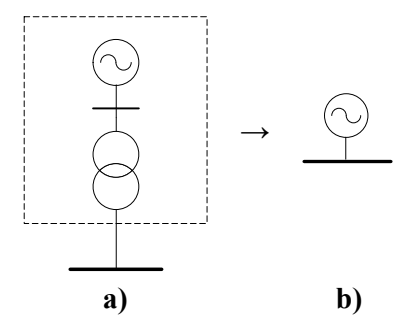

Figure 5. Generator/transformer aggregation representation.

Considering that both failure/repair cycle of the generator as the transformer follow the two-state Markov model, a multistate Markov model can be assumed and is represented in Fig. 6, where all possible states of operation are displayed.

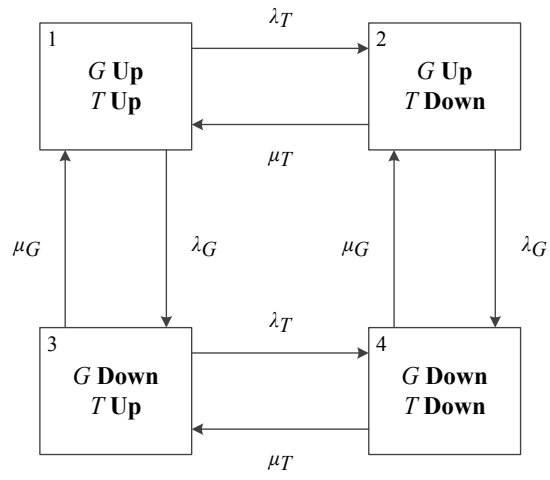

Figure 6. Multistate Markov model

( $\lambda_{G}$ and $\lambda_{T}$ are the failure rates of the generator and transformer respectively, $\mu_{G}$ and $\mu_{T}$ are the repair rate of the generator and transformer respectively).

Accepting this model, the generation capacity is only available when the generator and transformer are in operation (state 1 in Fig. 6). The remaining states result in a failure to provide the generation capacity. In analogy with the two-state Markov model, state 1 of the multistate Markov represented in Fig. 6 corresponds to the up state of the two-states Markov model depicted in Fig. 2 and the remaining states correspond to the down state. To transform the multistate Markov described in Fig. 6 it is necessary to calculate the parameters of the stochastic model of the two-state Markov equivalent model[1]. The probability for state 1 is given by

$$
P_{1}=P_{U p}=\frac{\mu_{G} \mu_{T}}{\left(\lambda_{G}+\mu_{G}\right)\left(\lambda_{T}+\mu_{T}\right)} .
$$


The probability $P_{1}$ corresponds to the probability of the up state, $P_{U p}$, of the two-state Markov model (Fig.2). The down state probability, $P_{\text {Down }}$, is given by $\mathrm{P}_{\text {Down }}=1-\mathrm{P}_{\mathrm{Up}}$. The equivalent failure rate, $\lambda_{S}$ and the equivalent repair rate, $\mu_{S}$ are calculated by

$$
\begin{gathered}
\lambda_{S}=\lambda_{G}+\lambda_{T} \\
\mu_{S}=\lambda_{S} \frac{P_{1}}{1-P_{1}} .
\end{gathered}
$$

\section{REPRESENTING THE INERTIAL LOAD}

As previously stated, it is a common practice to place first in the dispatch procedure a more stable set of generating units, such as thermal and hydro units, to fulfill the largest share of the load diagram. In order to represent this practice into the SMCS procedure for adequacy evaluation, a simple model was proposed in [6]. This model establishes that a specified amount of load, designated as inertial load, must always be provided by a fixed set of generation units regardless of the hourly load variation, ensuring the system stability and security. When this set of generators is not enough to attend the inertial load (e.g. due to unit failures), the inertial load is set to the maximum capacity available and the event is marked as an insecure state. Ultimately, the system stability and security cannot be ensured only by this restriction, depending also on the dynamic characteristics of certain generating units, dispatch and other procedures set by the system operator. However, the insecure state concept is presented to identify the events when the pre-established set of generation units is insufficient to attend the inertial load. Therefore, a set of new indices can be defined to estimate insecure state events [2] into the adequacy evaluation, which in turn can validate the adequacy evaluation:

- Insecure state probability (ISP): probability of an insecure state event.

- Insecure state expectation (ISE): average number of hours in which insecure states occur $(\mathrm{h} / \mathrm{yr})$.

- $\quad$ Expected inertial load not supplied (EILNS): expected power by which the set of generation units fail to supply the inertial load (MW).

- Expected inertial energy not supplied (EIENS): expected energy by which the set of generation units fail to supply the inertial load (MWh/yr).

- Insecure state frequency (ISF): average frequency of insecure state events (occ./yr).

- Insecure state duration (ISD): average duration of insecure state events (h/occ.).

The value of the inertial load and the set of units programed to attend it depend on several different characteristics of the system under evaluation. This concept of insecure state requires a careful analysis, providing valuable information about the effective adequacy evaluation performed.

\section{DEMONSTRATION CASE}

The experiments supporting this paper were performed on two demonstration cases: 1) first, on modified versions of the IEEE-RTS 79 [7] system and 2) in a real small scale system.

\section{A. IEEE-RTS $79 \mathrm{HW}$}

The well-known configuration of the IEEE-RTS 79 [7] consists of 32 generating units, 24 buses, 33 transmission lines, and 5 transformers. The installed capacity is $3405 \mathrm{MW}$, from which only $8.8 \%$ originates from renewable sources, corresponding to $300 \mathrm{MW}$ of hydro capacity. To account for the variability of renewable power in the system, a modification of the IEEE-RTS 79 is proposed. This new configuration is called IEEE-RTS $79 \mathrm{HW}$ and consists of adding wind power capacity to the original configuration. The additional wind farms aggregate units of $2 \mathrm{MW}$ $(\mathrm{MTTF}=1914.74 \mathrm{~h}$ and $\mathrm{a}$ MTTR $=80 \mathrm{~h})$ and are connected to buses 8,11 and 19. The wind farms have historical capacity time-dependent series defined on an hourly basis [5]. The set of units assuring the inertial constraint are the four $155 \mathrm{MW}$ units, the $350 \mathrm{MW}$ unit, and the two $400 \mathrm{MW}$ units of the original configuration of the IEEE-RTS 79 [7].

Table I presents the loss of load indices obtained for different values of wind capacity in the IEEE-RTS 79 HW.

TABLE I. LOSS OF LOAD INDICES FOR IEE-RTS $79 \mathrm{HW}$

\begin{tabular}{|c|c|c|c|c|}
\hline $\begin{array}{c}\text { IEEE-RTS } \\
\text { 79 HW }\end{array}$ & $\begin{array}{c}\text { LOLE } \\
\text { (h/yr) }\end{array}$ & $\begin{array}{c}\text { EENS } \\
\text { (MWh/yr) }\end{array}$ & $\begin{array}{c}\text { LOLF } \\
\text { (occ./yr) }\end{array}$ & $\begin{array}{c}\text { LOLD } \\
\text { (h/occ.) }\end{array}$ \\
\hline $400 \mathrm{MW}$ & 11.554 & 1435.02 & 2.436 & 4.743 \\
\hline $800 \mathrm{MW}$ & 6.581 & 802.87 & 1.391 & 4.730 \\
\hline $1200 \mathrm{MW}$ & 4.189 & 495.55 & 0.917 & 4.571 \\
\hline $1600 \mathrm{MW}$ & 2.832 & 328.58 & 0.629 & 4.499 \\
\hline
\end{tabular}

The results show that when renewable capacity is added to the system, the value of the loss of load indices decreases. The LOLE index for the configuration with $400 \mathrm{MW}$ of wind power is $11.554 \mathrm{~h} / \mathrm{yr}$ and this value is considerably reduce to $2.832 \mathrm{~h} / \mathrm{y}$ when $1600 \mathrm{MW}$ were added to the system. The value of EENS was also diminished when wind capacity was added to the system, from $1435 \mathrm{MWh} / \mathrm{yr}$ in the $400 \mathrm{MW}$ configuration to $329 \mathrm{MWh} / \mathrm{yr}$ in the $1600 \mathrm{MW}$ configuration. These results indicate a clear inverse relation between the loss of load indices and the increase of wind power capacity in the system, leading to conclude that the integration of renewable capacity (wind power in this case) leads to a more reliable system. However, despite the evidences shown in Table I, the global indices may hide security aspects, as previously stated in Section III.

Table II presents the insecure state indices and Fig. 7 presents the insecure state index ISE versus the loss of load index LOLE, obtained for the IEEE-RTS $79 \mathrm{HW}$.

TABLE II. INSECURE STATE INDICES FOR IEEE-RTS 79 HW

\begin{tabular}{|c|c|c|c|c|c|c|}
\hline $\begin{array}{c}\text { IEEE-RTS } \\
\text { 79 HW }\end{array}$ & $\begin{array}{c}\text { ISP } \\
\text { (prob.) }\end{array}$ & $\begin{array}{c}\text { ISE } \\
\text { (h/yr) }\end{array}$ & $\begin{array}{c}\text { EILNS } \\
\text { (MW) }\end{array}$ & $\begin{array}{c}\text { EIENS } \\
\text { (MWh/yr) }\end{array}$ & $\begin{array}{c}\text { ISF } \\
\text { (occ./yr) }\end{array}$ & $\begin{array}{c}\text { ISD } \\
\text { (h/occ.) }\end{array}$ \\
\hline $400 \mathrm{MW}$ & 0.0058 & 50.628 & 0.7598 & 6656.03 & 1.793 & 28.244 \\
\hline $800 \mathrm{MW}$ & 0.0057 & 49.996 & 0.7528 & 6594.46 & 1.792 & 27.895 \\
\hline $1200 \mathrm{MW}$ & 0.0058 & 50.941 & 0.7634 & 6687.44 & 1.785 & 28.538 \\
\hline $1600 \mathrm{MW}$ & 0.0056 & 49.296 & 0.7337 & 6427.06 & 1.756 & 28.065 \\
\hline
\end{tabular}




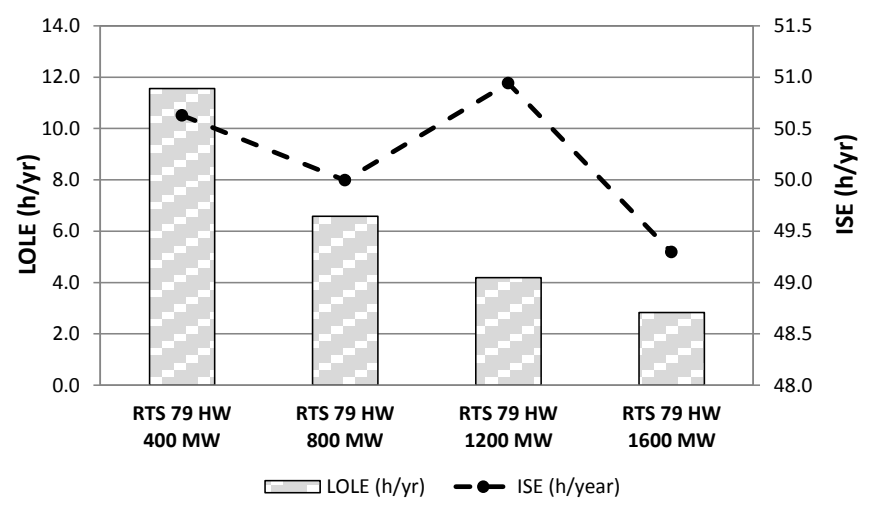

Figure 7. LOLE and ISE indices for IEEE-RTS $79 \mathrm{HW}$.

The insecure state indices aim to assist operators in evaluating the system considering security aspects into an adequacy evaluation. In this context, the ISE index estimates the number of hours in which insecure states occur. Analyzing Table II, one can see that despite the growth of generation capacity in the system, the occurrence of insecure state events remains in the narrow interval, where the ISE index ranges from 49 to $51 \mathrm{~h} / \mathrm{yr}$ for all wind power configurations.

This conclusion can be complemented with Fig. 7, where one can see that the decrease of the LOLE index is not followed by the ISE index. Looking closely to Fig. 7 one can notice that the LOLE index ranges from nearly $12 \mathrm{~h} / \mathrm{yr}$ for $400 \mathrm{MW}$ of wind power capacity and $2 \mathrm{~h} / \mathrm{yr}$ when the wind power capacity is increased to $1600 \mathrm{MW}$. This decrease is gradual and in direct proportion with the wind power capacity added to the system. This difference can be expressed through a variation $\triangle \mathrm{LOLE} \approx 12-3=9 \mathrm{~h} / \mathrm{yr}$. A similar analysis can be carried out for the ISE index where the difference between configurations results in a $\Delta \mathrm{ISE} \approx 51-49=2 \mathrm{~h} / \mathrm{yr}$. These results reveal that the increase of wind capacity in the system leads to a significant decrease in the LOLE index but in terms of security it only resulted in a 2 hour difference for the ISE index which is residual taking into account the magnitude scale of the correspondent index. Also the correlation between LOLE and ISE is not clear. The LOLE index decreases when wind power capacity is added, reflecting the robustness of the system in terms of generation capacity to attend the load, however the ISE index varies differently: it decreases when the wind power capacity is set at $800 \mathrm{MW}$, increases when the wind power capacity is at $1200 \mathrm{MW}$ and decreases again for the $1600 \mathrm{MW}$ configuration. The intuitive interpretation for this behavior is linked to the meshed network that characterizes the IEEE-RTS 79 HW system.

The results presented for the IEEE-RTS 79 HW system show that despite the system presents a robust performance from the adequacy point of view, the same evaluation cannot be highlighted when security aspects are introduced into the evaluation.

\section{B. Real Case Study}

The real system presented consists in a planned configuration, typically radial, with an installed capacity of around $500 \mathrm{MW}$ from which $40 \%$ is originated from renewable power units (Case Study 1). A variation of this system (Case Study 2) was also evaluated assuming a future configuration with a more representative share of renewable capacity to account the expected growth of load in the system. In this configuration the wind power capacity is increased by a factor of 2 (doubled). The grid is spread with new buses and transmissions circuits to connect the new wind farms. The system load is increased by a factor of 1.5 , compared with Case Study 1, and the inertial load value is re-defined by the same factor of 1.5. The set of units assuring the inertial constraint is maintained.

In both case studies the hydro and wind capacity varies hourly according with historical series (as in Fig. 4). The generators stochastic parameters (MTTF and MTTR) were obtained from statistical data bases and literature review, considering similarities in terms of technology and power capacity [8].

For every case study a probability analysis was performed in order to assess the impact of the integration of renewable power in the system adequacy. Table I presents the loss of load indices obtained for the two case studies.

TABLE III. LOSS OF LOAD INDICES FOR THE REAL CASE STUdY

\begin{tabular}{|c|c|c|c|c|}
\hline $\begin{array}{c}\text { Real Case } \\
\text { Study }\end{array}$ & $\begin{array}{c}\text { LOLE } \\
\text { (h/yr) }\end{array}$ & $\begin{array}{c}\text { EENS } \\
\text { (MWh/yr) }\end{array}$ & $\begin{array}{c}\text { LOLF } \\
\text { (occ./yr) }\end{array}$ & $\begin{array}{c}\text { LOLD } \\
\text { (h/occ.) }\end{array}$ \\
\hline Case Study 1 & 5.731 & 37.85 & 1.557 & 3.668 \\
\hline Case Study 2 & 0.950 & 3.82 & 0.312 & 3.043 \\
\hline
\end{tabular}

From this analysis, regarding Case Study 1, the LOLE index estimated was $5.731 \mathrm{~h} / \mathrm{yr}$, while in Case Study 2 the index estimation was $0.950 \mathrm{~h} / \mathrm{yr}$. The loss of load frequency (LOLF) estimated in Case Study 1 was 1.557 occ./yr while in Case Study 2 the value estimated was 0.312 occ./yr. The first conclusion extracted from this evaluation is that the integration of more renewable capacity in the system, to face the increase of load, leads to a more reliable system, where loss of load events are rare (like in the IEEE-RTS 79 HW system). Once again the global indices may neglect dynamic security concerns.

For a detailed analyses regarding security aspects, Table IV presents the insecure state estimates and Fig. 8 compares the ISE index with the loss of load expectation index (LOLE), obtained for the two case studies.

\section{TABLE IV. InSECURE StATE INDICES For REAL CASE StUdy}

\begin{tabular}{|c|c|c|c|c|c|c|}
\hline $\begin{array}{c}\text { Real Case } \\
\text { Study }\end{array}$ & $\begin{array}{c}\text { ISP } \\
\text { (prob.) }\end{array}$ & $\begin{array}{c}\text { ISE } \\
\text { (h/yr) }\end{array}$ & $\begin{array}{c}\text { EILNS } \\
\text { (MW) }\end{array}$ & $\begin{array}{c}\text { EIENS } \\
\text { (MWh/yr) }\end{array}$ & $\begin{array}{c}\text { ISF } \\
\text { (occ./yr) }\end{array}$ & $\begin{array}{c}\text { ISD } \\
\text { (h/occ.) }\end{array}$ \\
\hline Case Study 1 & 0.0010 & 8.592 & 0.0050 & 43.51 & 0.225 & 38.207 \\
\hline Case Study 2 & 0.0182 & 159.004 & 0.1337 & 1170.76 & 2.954 & 53.825 \\
\hline
\end{tabular}

The ISE index was estimated in $8.592 \mathrm{hr} / \mathrm{yr}$ for Case Study 1 and $159.004 \mathrm{hr} / \mathrm{yr}$ in Case Study 2. The ISF index, which lists the average frequency of an insecure state event per year, was also listed and for Case Study 1 it was estimated in $0.225 \mathrm{occ} . / \mathrm{yr}$ and for Case Study 2 this estimation increases almost to $3 \mathrm{occ}$./yr. Note that the inertial load was increase in Case Study 2, to account the increase of renewable power in the system. However, the integration of renewable capacity and the decrease in the use of conventional generation lead to a degradation of all security indexes listed in Table IV. 


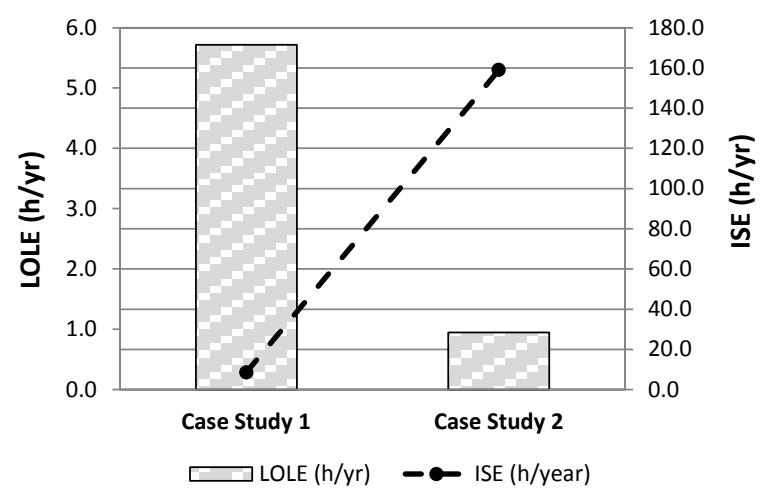

Figure 8. LOLE and ISE indices for the Real Case Study.

This analysis can be supported observing Fig. 8 and measuring the differences in the indexes depicted. In Case Study 1, the LOLE index decreases when wind capacity is added to the system, expressed by the difference $\Delta \mathrm{LOLE} \approx 6-1=5 \mathrm{~h} / \mathrm{yr}$. Regarding the insecure evaluation, in Case Study 1, the ISE index achieved was around $10 \mathrm{~h} / \mathrm{yr}$, whereas in the Case Study 2, the ISE index achieved was around $160 \mathrm{~h} / \mathrm{yr}$, resulting in a $\Delta \mathrm{ISE} \approx 160-10=150 \mathrm{~h} / \mathrm{yr}$. After examining these results, one can see that the integration of more wind power capacity in the system results in the decrease of loss of load events, in similarity with the IEEERTS 79 HW system. However in Case Study 2, despite the growth in generation capacity, when the inertial load increases, the occurrence of insecure state events becomes more frequent, witnessed by the increase of the ISE index. In fact, the $\triangle \mathrm{ISE}$ of $150 \mathrm{~h} / \mathrm{yr}$ represents a massive growth of insecure events and, once again, the radial configuration of this system can explain this substantial growth of insecure events, due to the lack of options to drain the energy throughout the system.

The results obtain for this case study show that the integration of renewable capacity, can improve the adequacy evaluation but, if neglected, it can also weaken the system performance from the security point of view.

\section{CONCLUSIONS}

This paper presents an adequacy assessment of a composite system to identify the impact of massive integration of renewable sources in the system reliability. When new renewable units are integrated in the system they will influence the dynamic behavior of the system and, consequently, the value of the inertial load. The major issue concerning this study was to demonstrate that the integration of renewable power in a system, may keep the global indices within safety levels, but can bring additional risks to the system stability by increasing the number of events where the inertial load was not met. The value of the inertial load, the units capable of supplying it and the configuration of the grid depends on the system, and can result in different uses of the generation resources. The demonstration case revealed that the configuration nature of the system can influence the security performance. If the system is markedly radial, the flow of energy will be constrained by the lack of options in the grid which may result in the increase of events where the inertial load was not attended, therefore jeopardizing the system security, even though the loss of load events is not directly affected.

The results obtained from the demonstration cases illustrate that the integration of renewable capacity should be carefully thought from the adequacy point of view without neglecting the stability concerns, reaffirming the need to integrate and combine adequacy and security aspects into power system analysis.

\section{ACKNOWLEDGMENT}

The authors gratefully acknowledge FCT - Fundação para a Ciência e a Tecnologia (Portuguese Foundation for Science and Technology) for the financial support through Ph.D Scholarships SFRH/BD/43004/2008，SFRH/BD/43049/2008 and "Ciência 2008" program.

\section{REFERENCES}

[1] R. N. Allan, and R. Billinton, "Reliability Evaluation of Power Systems", 2nd ed., New York: Plenum Press, 1996.

[2] L. M. Carvalho, M. A. da Rosa, A. M. Leite da Silva, and V. Miranda, "Probabilistic analysis for maximizing the grid integration of wind power generation," IEEE Transactions on Power Systems, in press.

[3] R. Billinton and W. Li, Reliability Assessment of Electric Power Systems Using Monte Carlo Methods. New York: Plenum, 1994.

[4] R. Billinton, G. Yi, and R. Karki, "Composite system adequacy assessment incorporating large-scale wind energy conversion systems considering wind speed correlation," IEEE Trans. Power Syst., vol. 24, no. 3, pp. 1375-1382, Aug. 2009.

[5] A. M. Leite da Silva, W. S. Sales, L. A. da Fonseca Manso, and R. Billinton, "Long-term probabilistic evaluation of operating reserve requirements with renewable sources," IEEE Trans. Power Syst., vol. 25, no. 1, pp. 106-116, Feb. 2010.

[6] L. M. Carvalho, M. A. da Rosa, V. Miranda, and R. Ferreira, "A chronological composite system adequacy assessment considering nondispatchable renewable energy sources and their integration strategies," in Proc. 17th PSCC, Stockholm, Sweden, Aug. 22-26, 2011.

[7] IEEE Reliability Test System Task Force of the Application of Probability Methods Subcommittee, "IEEE reliability test system", IEEE Transactions on PAS, vol. PAS-98, no. 6, pp. 2047-2054, Nov./Dec. 1979.

[8] North American Electric Reliability Corporation (NERC), Generating Availability Data System (GADS). Available: http://www.nerc.com/page.php?cid=4\%7C43 